\title{
Improved Bounds on Maximum Size Binary Radar Arrays
}

\author{
Jon Hamkins ${ }^{1}$ \\ Jet Propulsion Laboratory \\ 4800 Oak Grove Drive \\ Pasadena, CA 91109-8099 \\ email: hamkins@jpl.nasa.gov
}

\author{
Kenneth Zeger ${ }^{1}$ \\ Department of Electrical and Computer Engineering \\ University of California, San Diego \\ La Jolla, CA 92093-0407 \\ email: zeger@ucsd.edu
}

\begin{tabular}{|c|c|c|c|c|}
\hline \multirow{2}{*}{$N$} & \multicolumn{4}{|c|}{$\mathrm{G}(\mathrm{N})$} \\
\cline { 2 - 5 } & $\begin{array}{c}\text { old } \\
\text { lower } \\
\text { bound }\end{array}$ & $\begin{array}{c}\text { new } \\
\text { lower } \\
\text { bound }\end{array}$ & $\begin{array}{c}\text { new } \\
\text { upper } \\
\text { bound }\end{array}$ & $\begin{array}{c}\text { old } \\
\text { upper } \\
\text { bound }\end{array}$ \\
\hline 9 & 23 & 24 & & 24 \\
10 & 26 & & 26 & 27 \\
11 & 29 & & 29 & 30 \\
12 & 32 & & 32 & 33 \\
13 & 34 & 35 & & 35 \\
14 & 37 & & 37 & 38 \\
15 & 40 & & 40 & 41 \\
16 & 42 & 43 & & 44 \\
17 & 45 & 46 & & 47 \\
\hline
\end{tabular}

Abstract - We determine the maximum size of radar arrays containing 9-15 rows, and for those containing 16 and 17 rows we narrow the maximum size down to two values. We also give improved upper and lower asymptotic bounds on the maximum size of radar arrays, which narrow the gap between the existing upper and lower asymptotic bounds by more than $25 \%$.

\section{INTRODUCTION}

A radar array is an $N \times M$ binary matrix, such that every column contains exactly one "1", and such that the horizontal autocorrelation function can only take on the values 0,1 , and $M[1,2]$. That is, the 1 's of a horizontally (time) shifted version of the array overlap 1's of the unshifted array at most one time. Let $G(N)$ be the maximum value for which an $N \times G(N)$ radar array exists. The radar array problem is to determine $G(N)$. This is currently an unsolved problem, although some bounds have been obtained in the past by several researchers.

We may regard a radar array as an $N \times M$ grid with one "dot" per column, expressed by a vector $\left(r_{1}, \ldots, r_{M}\right)$, where for each $i$, the integer $r_{i}$ indicates which row contains the dot of the $i$ th column. Whenever $r_{i}=r_{j}$, the distance $|i-j|$ is called the spacing of the pair $(i, j)$. Note that a binary matrix with exactly one " 1 " per column is a radar array if and only if each positive spacing appears at most once.

The precise asymptotic behavior of $G(N)$ is not presently known. The tightest previously known bounds are $\frac{306}{113} \leq$ $\lim \sup _{N \rightarrow \infty} \frac{G(N)}{N} \leq \frac{9+\sqrt{5}}{4}$ [4]. There is a gap of 0.101 between the bounds.

\section{Radar Array Search Algorithm}

The number of $N \times M$ arrays that have 1 dot per column is $N^{M}$, a prohibitively large number for exhaustive computer searches. Thus, effective heuristic searching techniques are important if they produce new radar arrays. Most radar arrays known to be optimal have the following characteristics:

1. All rows have either 2 dots or 3 dots.

2. The spacings present in the radar array are all the integers in the range 1 to $2 M-3 N$.

By restricting attention to this type of radar array, a reduced complexity search yielded four new radar array sizes. By taking advantage of certain radar array properties, a reduced complexity search is possible for upper bounds as well. Table 1 summarizes the improvements made in the best known bounds on small radar arrays, using the algorithm we developed.

\footnotetext{
${ }^{1}$ This research was supported in part by the National Science Foundation and Engineering Research Associates.
}

Table 1: Improved upper and lower bounds on $\mathrm{G}(\mathrm{N})$.

\begin{tabular}{|l|l|}
\hline$N \times M$ & \multicolumn{1}{|c|}{ Row Location of dot in each column } \\
\hline $9 \times 24$ & {$[1-8] 7439958265142137$} \\
\hline $13 \times 35$ & {$[1-10] 9118612313137412115210$} \\
& 5814721369 \\
\hline $16 \times 43$ & {$[1-13] 7141512816410516915133$} \\
& 61411693528211471012 \\
\hline $17 \times 46$ & {$[1-14] 8151276161717163104159$} \\
& 1413259110571242136811 \\
\hline
\end{tabular}

Table 2: Four new radar arrays, in vector notation.

\section{NEW ASYMPTOTIC BOUNDS}

Theorem $1 \frac{276}{101} \leq \lim \sup _{N \rightarrow \infty} \frac{G(N)}{N} \leq \frac{20+\sqrt{6}}{8}$.

The proof of the upper bound uses a slightly stronger version of the window method in [4], by exploiting the fact that in any $N \times M$ array which has either 1 or 2 dots per row and 0 or 1 dots per column, with $M \geq 2 N$, at least $N / 4$ of the spacings $M-N, \ldots, M-1$ are not present in the array.

\section{REFERENCES}

[1] S. W. Golomb and H. Taylor, "Two-dimensional synchronization patterns for minimum ambiguity," IEEE Transactions on Information Theory, vol. IT-28, pp. 600-604, July 1982.

[2] J. P. Robinson, "Golomb rectangles," IEEE Transactions on Information Theory, vol. IT-31, pp. 781-787, Nov. 1985.

[3] A. Blockhuis and H. J. Tiersma, "Bounds for the size of radar arrays," IEEE Transactions on Information Theory, vol. 34, pp. 164-167, Jan. 1988.

[4] Z. Zhang and C. Tu, "New bounds for the sizes of radar arrays," IEEE Transactions on Information Theory, vol. 40, pp. 16721678. Sept. 1994. 